\title{
Padrão de Metadados para Objetos de Aprendizagem Baseado na Interação
}

\author{
Bruno N. Luz ${ }^{1,2}$, Rafael Santos ${ }^{2}$, Valéria F. Martins ${ }^{3}$, Marcelo P. Guimarães ${ }^{1,4}$ \\ ${ }^{1}$ Campus Boituva - Instituto Federal de São Paulo (IFSP) - Boituva - SP - Brasil \\ ${ }^{2}$ Programa de Mestrado em Ciência da Computação - Faculdade Campo Limpo Paulista \\ (FACCAMP) - Campo Limpo Paulista - SP - Brasil \\ ${ }^{3}$ Faculdade de Computação e Informática - Mackenzie - São Paulo - Brasil \\ ${ }^{4}$ Universidade Federal de São Paulo (UNIFESP) - São Paulo - Brasil \\ nogueira_luz@ifsp.edu.br, rafaelerenovaci.com, valfarinazzo@gmail.com, \\ marcelodepaiva@gmail.com
}

\begin{abstract}
Learning objects are designed to facilitate the creation and reuse of educational content, although they do not provide resources for teachers to identify and address students' difficulties. This paper aims to present a learning object model that increases the level of interaction between teachers and students to improve the learning process. This standard was based on SCORM and implemented in eTutor tool. The results of using this model are also presented.

Resumo. Objetos de Aprendizagem visam facilitar a criação e a reutilização de conteúdos educacionais, porém não fornecem recursos para que as dificuldades dos alunos sejam identificadas e tratadas pelos professores. Este artigo apresenta um modelo de padrão de objetos de aprendizagem que explora a interatividade entre alunos e professores para auxiliar o processo de ensino. Esse padrão foi derivado do SCORM e implementado na ferramenta eTutor. Os resultados de uso desse padrão são também apresentados.
\end{abstract}

\section{Introdução}

A evolução das Tecnologias da Informação e Comunicação (TICs) nos últimos anos tem permitido que os professores mantenham contato com os alunos, e estes com o restante do grupo, sem a limitação física de uma sala de aula tradicional. Contudo, o seu uso é ainda um desafio para muitos. Por exemplo, Hodgins (2000) e Willey (2000) relatam que muitos professores não conseguem criar Objetos de Aprendizagem (OA) de forma aprofundada porque não dominam o uso de tecnologias computacionais.

Segundo Willey (2000), os OA são entidades digitais, ou não, que podem ser utilizadas, reutilizadas e referenciadas durante o processo de ensino. Rodríguez e Ayala (2012) definem os OA como entidades digitais e interativas criadas para o processo de ensino e aprendizagem e geração de conhecimento. Este trabalho tem como objetivo apresentar os Objetos de Aprendizagem Interativos (OAI), que incorporam nos OA a interatividade entre os alunos e professores. Com esse recurso, o professor é capaz de acompanhar a aprendizagem dos alunos e realizar as intervenções quando necessárias. Esse padrão foi implementado no eTutor, que é um ambiente de ensino-aprendizagem. 
Kemezinski et.al (2012) descrevem uma metodologia para a construção de OAI, porém não se preocupam em estruturá-los conforme um padrão. Marczal e Direne (2012) também desenvolveram uma ferramenta de autoria baseada em OA que promove a interação entre: professor e $\mathrm{AO}$, e aluno e OA. Porém, não atendem as demandas apresentadas neste trabalho, que são as de garantir a interação ativa e efetiva entre o aluno e professor, mediado por um ambiente de aprendizagem.

O restante do artigo está organizado da seguinte forma: a seção 2 mostra uma visão geral sobre os principais OA; a seção 3 apresenta os OAI; os resultados dos testes realizados com os OAI são apresentados na seção 4; e, por fim, a seção 5 mostra as conclusões.

\section{Padrões para Objetos de Aprendizagem}

Os padrões para criação de OA devem garantir quatro características principais: interoperabilidade, reutilização, acessibilidade e durabilidade [Jordão, 2009 e Willey, 2000]. Para atender essas características, eles dependem principalmente de um conjunto de metadados que possam acompanhar e dar significados ao seu conteúdo instrucional.

Segundo Ferlin, Kemezinski, Murakami e Hounsell (2010), os principais padrões de metadados utilizados para a construção de OA são: Dublin Core, LOM/IMS-LD, ARIADNE e CanCore. Sendo que desses, o ARIADNE está relacionado diretamente com os Repositórios Digitais de OA. Vicari et. Al (2010) citam o OBAA que é um padrão que possibilita criar a partir do LOM um conjunto de metadados capaz de garantir seu uso em diversas plataformas, como TV Digital e dispositivos móveis. Existe também o SCORM, que vai além de um padrão de metadados, pois fornece um nível maior de documentação do que os outros, além de englobar uma coleção de especificações e normas que definem a inter-relação de objetos de conteúdo, modelos de dados e protocolos [ADL, 2009]. Porém, apresenta como restrição o foco na autoaprendizagem do aluno, sem a interferência de outros atores [Vahldick e Raabe, 2008].

A Tabela 1 mostra um comparativo entre os elementos dos principais padrões de metadados que permitem a criação de $\mathrm{OA}$. O atributo " $\mathrm{S}$ " indica que o padrão tem o elemento. O atributo "O" que é opcional. Segundo Ferlin, Kemezinski, Murakami e Hounsell (2010), esses elementos são encontrados em todos os padrões, o que faz deles essenciais para um padrão.

Tabela 1. Classificação dos Metadados Comuns em todos os Padrões Adaptado de Ferlin et. Al. (2010)

\begin{tabular}{c|c|c|c|c|c}
\hline \multicolumn{2}{c|}{ Padrões } & $\begin{array}{c}\text { LOM e } \\
\text { IMS-LD }\end{array}$ & $\begin{array}{c}\text { Can } \\
\text { Core }\end{array}$ & $\begin{array}{c}\text { Dublin } \\
\text { Core }\end{array}$ & SCORM \\
\hline \multirow{3}{*}{ Geral } & Metadados & $\mathrm{S}$ & $\mathrm{S}$ & $\mathrm{S}$ & $\mathrm{S}$ \\
\cline { 2 - 6 } & Entrada & $\mathrm{S}$ & $\mathrm{S}$ & $\mathrm{S}$ & $\mathrm{S}$ \\
\cline { 2 - 6 } & Título & $\mathrm{S}$ & $\mathrm{S}$ & $\mathrm{S}$ & $\mathrm{O}$ \\
\cline { 2 - 6 } & Idioma & $\mathrm{S}$ & $\mathrm{S}$ & $\mathrm{S}$ & $\mathrm{S}$ \\
\hline \multirow{2}{*}{ Ciclo de Vida } & Descrição & $\mathrm{S}$ & $\mathrm{S}$ & $\mathrm{S}$ & $\mathrm{O}$ \\
\cline { 2 - 6 } & Entidade & $\mathrm{S}$ & $\mathrm{S}$ & $\mathrm{S}$ & $\mathrm{O}$ \\
\hline Técnico & Data & $\mathrm{O}$ & $\mathrm{S}$ & $\mathrm{S}$ & $\mathrm{O}$ \\
\hline Educacional & Tipo de Recurso Aprendizagem & $\mathrm{S}$ & $\mathrm{S}$ & $\mathrm{S}$ & $\mathrm{O}$ \\
\hline Direitos & Formato & \multicolumn{2}{|c}{ Legenda: Sim(S) Opcional (O) }
\end{tabular}




\section{Padrão de Objetos de Aprendizagem Baseado na Interação}

Os recursos incorporados pelos OAI (Objetos de Aprendizagem Interativos) visam promover a interação entre: professor e aprendiz, aprendiz e professor, objeto e aprendiz, e objeto e professor. Como consequência, tornou-se necessário especificar um novo padrão de metadados.

A promoção da interação entre os envolvidos no processo de ensinoaprendizagem é potencializada por intermédio, principalmente, da notificação do estado atual de aprendizagem do aluno para o professor, que recebe informações, como, por exemplo, "o aluno está por volta de 5 minutos sem interagir com o objeto". A percepção do estado de aprendizagem dos alunos pode ocorrer via mensagens de texto ou imagens. No caso do eTutor, a interface do professor utiliza-se de cores nas imagens dos alunos para notificar os professores.

Além disso, o professor conta com ferramentas agregadas ao contexto que facilitam a comunicação com o aluno (por exemplo, chat) e que fornecem conteúdos conforme a situação do aluno. Os OAI possuem parâmetros que permitem a configuração dos mesmos de tal maneira que, por exemplo, se o aluno passar mais de 3 minutos sem interagir com o mesmo, dicas são oferecidas, que podem ser vídeos, apresentações ou textos (Figura 1). Dessa forma, os objetos são capazes de instigar os alunos de forma automática, estimulando uma aprendizagem independente, contudo acompanhada pelo professor.

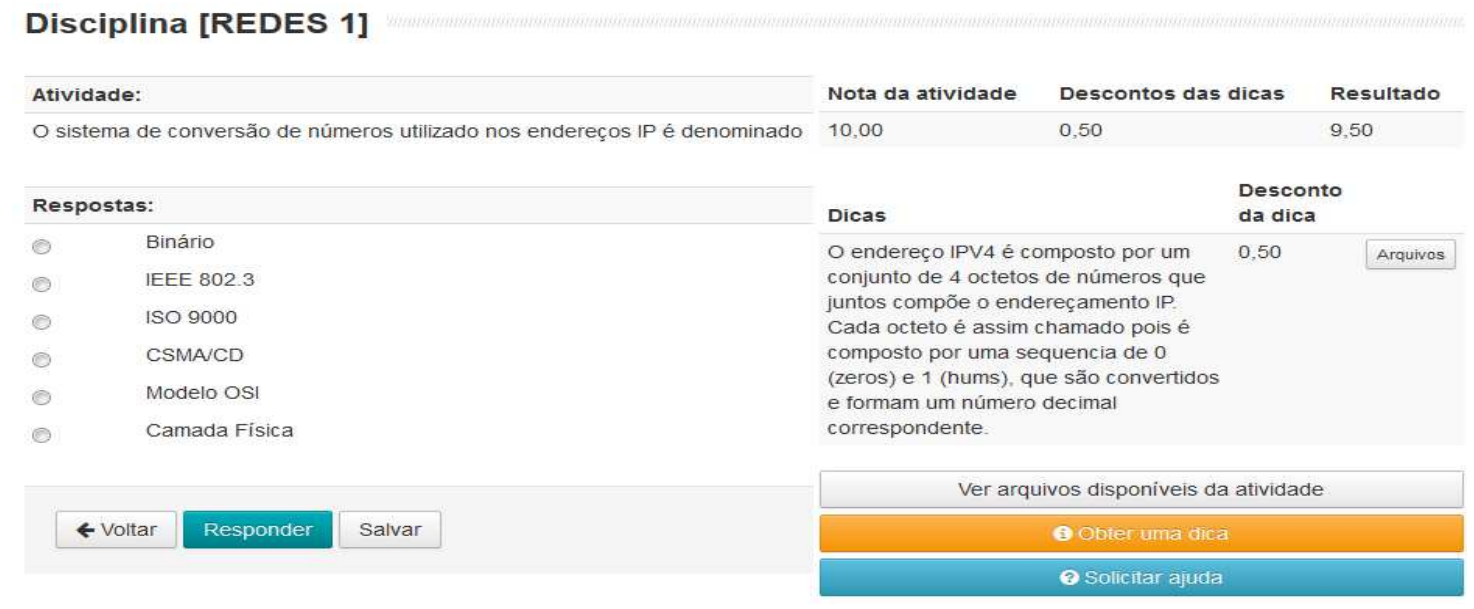

Figura 1. OAl disponível no eTutor.

Ao interagir automaticamente com o aluno, os OAI notificam o professor com avisos e informações sobre a atividade em questão, permitindo assim o acompanhamento individualizado de cada aluno pelo professor. Situações como essa de alerta ao professor podem caracterizar dificuldades do aluno na execução da atividade. Com o conhecimento da situação no momento real, o professor pode realizar a ação apropriada. Estes recursos foram implementados no ambiente eTutor.

A Figura 2 mostra as adaptações implementadas no padrão de metadados do SCORM para atender os requisitos dos OAI, o que resultou em 55 elementos. O padrão SCORM foi escolhido como base porque contém os elementos comuns aos principais padrões, que são majoritariamente opcionais. 


\begin{tabular}{|c|c|c|c|c|}
\hline \multicolumn{5}{|l|}{ CLASS BROWSER } \\
\hline \multicolumn{5}{|l|}{ For Proiect: $\bullet$ OAl } \\
\hline \multicolumn{4}{|r|}{ S. $\% \div \div=$} & \\
\hline - OAl & १ ○ 3. Direitos & - 5.2.2. Nivel & $9 \bigcirc 5.4 .7 .5$. Anexo & \\
\hline P ○ 1. Geral & - 3.1. Contato & - 5.2.3. Aspectos & - 5.4.7.5.1. Descriçẫo & \\
\hline 1.1 - Identific ador & 3.2. Autor & 5.3. Tipo Recurso & - 5.4.7.5.2. Formato & \\
\hline 1.2- Título & - 3.3. Licença & १ 5.4. Atividade & - 5.4.7.5.3. Tamanho & \\
\hline - 1.3 - Idioma & ९ ○ 4. Técnico & - 5.4.1. Identificador & P 5.4.8. Avaliaçắo & \\
\hline 1.4 - Sigla & - 4.1. Formato & - 5.4.2. Descriçẵo & - 5.4.8.1. Valor & \\
\hline - 1.5 -Numero_Atividades & - 4.2. Localizaçäo & 5.4.3. Ordem & - 5.4.8.2. Correçẵo & \\
\hline - 1.6 - Descriçẵo & - 4.3. Observaçốes Instalaçẵo & 5.4.4. Tipo & - 5.4.9. Dificuldade & \\
\hline i $\circ$ 2. Ciclo de Vida & - 4.4. Requisitos & - 5.4.5. Interaçắo Tipo & & \\
\hline 2.1. Versão & - 4.5. Plataforma Suportada & - 5.4.6. Ação Interaçã̃o & & \\
\hline - 2.2. Status & 4.6. Recursos Especifícos Plataforme & $P \multimap$ 5.4.7. Dicas & & \\
\hline १ 2.3. Contribuiçẳo & ค ○ 5. Educacional & 5.4.7.1. ID & & \\
\hline - 2.3.1. Função & - 5.1. Contexto & - 5.4.7.2. Descriçăo & & \\
\hline - 2.3.2. Entidade & ९ • 5.2. Interaçẵo & - 5.4.7.3. Desconto & & \\
\hline 2.3.3. Data & - 5.2.1. Percepção & 5.4.7.4. Tempo Dica & & \\
\hline
\end{tabular}

Figura 2. Elementos do Padrão OAl.

\section{Metodologia, Discussão e Resultados}

O suporte aos OAI foi implementado no ambiente de aprendizagem eTutor. Essa ferramenta foi desenvolvida especificamente para esse fim. Os testes desses objetos foram realizados por um professor ao ministrar o mesmo curso (16 horas) para duas turmas distintas, sendo que a Turma A utilizou os OAI e a Turma B não. As turmas tinham respectivamente cinco e quatro alunos. Após os testes, os alunos da Turma A responderam um questionário sobre a interação (5- "excelente", 4-“ótimo",3-"bom", 2-"regular" e 1"pouca influência") (Tabela 2). As notas finais da Turma B também foram utilizadas para efeito de comparação e validação dos OAI. As aulas da Turma A foram ministradas em laboratório, sendo que toda a interação entre os envolvidos foi síncrona.

Tabela 2. Respostas do questionário dos alunos referentes a interação.

\begin{tabular}{|c|c|c|c|c|c|}
\hline ALUNOS & A1 & A2 & $\mathbf{A 3}$ & A4 & A5 \\
\hline Acompanhamento do professor durante a atividade. & 5 & 4 & 4 & 4 & 5 \\
\hline $\begin{array}{l}\text { Durante a utilização do objeto de aprendizagem, você interagiu com o } \\
\text { professor solicitando ajuda? }\end{array}$ & Não & Não & Sim & Sim & Sim \\
\hline $\begin{array}{l}\text { Durante a utilização do objeto de aprendizagem, o professor acompanhou a } \\
\text { sua atividade? }\end{array}$ & Sim & Sim & Sim & Sim & Sim \\
\hline Possibilidade de solicitar e receber ajuda do professor durante a atividade. & 3 & 3 & 5 & 5 & 5 \\
\hline $\begin{array}{l}\text { Possibilidade de acesso aos arquivos disponibilizados junto com as dicas com } \\
\text { intuito de auxiliar na atividade. }\end{array}$ & 4 & 1 & 5 & 4 & 4 \\
\hline Possibilidade de interação no Chat com o professor durante a atividade. & 3 & 3 & 4 & 4 & 4 \\
\hline
\end{tabular}

Apenas $40 \%$ dos alunos não interagiram com o professor solicitando auxílio. Destaca-se também $40 \%$ dos alunos indicam o acompanhamento do professor durante as atividades como excelente e $60 \%$ avaliaram como ótima esta ação. Os testes aplicados também apresentaram resultados positivos ao se comparar as médias das notas das duas turmas, com uma diferença de 1,3\% maior no uso do OAI (Tabela 3).

Tabela 3. Resultado com as Notas dos Alunos.

\begin{tabular}{|c|c|c|c|c|c|c|c|c|c|c|c|}
\hline \multicolumn{7}{|c|}{ TURMA A - com OAI } & \multicolumn{5}{|c|}{ TURMA B -sem OAI } \\
\hline ALUNOS & A1 & A2 & A3 & A4 & A5 & Média & A1 & A2 & $\mathbf{A 3}$ & A4 & Média \\
\hline NOTAS & 7 & 9 & 8 & 7 & 8 & 7,8 & 6 & 6 & 7 & 7 & 6,5 \\
\hline
\end{tabular}

O professor avaliou o uso dos OAI como excelente, relatando que a ferramenta atende a que as características propostas pelo padrão. Além disso, destacou a facilidade de criação dos objetos no eTutor. 


\section{Conclusão}

Os OAI visam potencializar, principalmente, a relação aluno-professor, o que pode resultar em ganhos educacionais. Além disso, como os OAI são fundamentados nos princípios de Objetos de Aprendizagem, então agregam também características como: acessibilidade, reusabilidade, interoperabilidade e durabilidade.

Os testes realizados mostraram resultados positivos, indicando, principalmente, que o padrão proposto é capaz de potencializar a interação entre os envolvidos. Contudo, como a extensão dos testes foi limitada, existe ainda demanda por mais investigações para que os OAI sejam melhor validados. Além disso, existe a necessidade de testes que englobem a interação de maneira assíncrona.

\section{Referências}

ADL (2009) "SCORM 2004 4th Edition. Content Aggregation Model [CAM]. Version 1.1. August 14. Disponível em: <http://www.adlnet.gov/wp-content/uploads/ 2011/07/SCORM_2004_4ED_v1_1_Doc_Suite.zip>. Acesso em: 17/07/14.

Ferlin, J., Kemezinski, A., Murakami, E., Hounsell, M. S. (2010) "Metadados Essenciais: Uma metodologia para Catalogação de Objetos de Aprendizagem no Repositório Digital ROAI”. In: Anais do XVI Workshop Sobre Informática na Escola WIE 2010. Belo Horizonte-MG.

Hodgins, H. W. (2000) “The future of learning objects". In D. A. Wiley (Ed.), The Instructional Use of Learning Objects: Online Version. Retrieved MONTH DAY, YEAR, from the World Wide Web: 〈http://reusability.org/read/chapters/hodgins.doc>

Kemezinski, A, Costa, I. A., Wehrmeister, M. A., Hounsell, M. S. e Vahldick, A. (2012) "Metodologia para Construção de Objetos de Aprendizagem Interativos". In: Anais do $23^{\circ}$ Simpósio Brasileiro de Informática na Educação (SBIE 2012). Rio de Janeiro-RJ.

Marczal, D. e Direne, A. (2012) "FARMA: Uma ferramenta de autoria para objetos de aprendizagem de conceitos matemáticos". In: Anais do $23^{\circ}$ Simpósio Brasileiro de Informática na Educação (SBIE 2012). Rio de Janeiro-RJ.

Rodríguez, V. e Ayala, G. (2012) "Adaptivity and Adaptability of Learning Objetct's Interface". In: International Journal of Computer Applications. Vol. 37, No 1, January.

Vahldick, A., Raabe, A. L. A. (2008) “Adaptação de Conteúdo SCORM em Ambientes Inteligentes de Aprendizagem”. In: Anais do XIX Simpósio Brasileiro de Informática na Educação (SBIE 2008), Fortaleza-CE.

Vicari, R. M., Bez, M., Silva, J. M. C., Ribeiro, A., Gluz, J. C., Passerino, L., Santos, E., Primo, T.T, Rossi, L, H. L., Bordignon, A., Behar, P., Filho, R., Roesler, V. (2010) "Proposta Brasileira de Metadados para Objetos de Aprendizagem Baseados em Agentes (OBAA)". In: Revista Renote v.8, n². CINTED-UFRGS.

Wiley, D. A. (2000) "Connecting learning objects to instructional design theory: A definition, a metaphor, and a taxonomy". In D. A. Wiley (Ed.), The Instructional Use of Learning Objects: Online Version. Retrieved MONTH DAY, YEAR, from the World Wide Web: http://reusability.org/read/chapters/wiley.doc. 